\title{
A preoperative nomogram to predict the risk of synchronous distant metastases at diagnosis of primary breast cancer
}

\author{
C Boutros ${ }^{1}$, C Mazouni ${ }^{\star, 2}$, F Lerebours ${ }^{3}$, D Stevens ${ }^{3}$, X Lei $^{4}$, A M Gonzalez-Angulo ${ }^{5}$ and S Delaloge ${ }^{1}$ \\ ${ }^{1}$ Department of Breast and Medical Oncology, Institut Gustave Roussy, Villejuif 94805, France; ${ }^{2}$ Department of Surgery, Division of \\ Breast and Plastic Surgery, Institut Gustave Roussy, Villejuif 94805, France; ${ }^{3}$ Department of Breast and Medical Oncology, Institut \\ Curie, Hôpital René Huguenin, Saint-Cloud, France; ${ }^{4}$ Department of Biostatistics, MD Anderson Cancer Center, Houston, TX, USA \\ and ${ }^{5}$ Department of Breast Medical Oncology, University of Texas, MD Anderson Cancer Center, Houston, TX, USA
}

Background: The detection of synchronous metastases at primary diagnosis of breast cancer (BC) affects its initial management. A risk calculator that incorporates many factors to evaluate an individual's risk of harbouring synchronous metastases would be useful to adapt cancer management.

Patients and Methods: Patients with primary diagnosis of BC were identified from three institutional databases sharing homogeneous work-up recommendations. A risk score for synchronous metastases was estimated and a nomogram was constructed using the first database. Its performance was assessed by receiver characteristic (ROC) analysis. The nomogram was externally validated in the two independent cohorts.

Results: A preoperative nomogram based on the clinical tumour size $(P<0.001)$, clinical nodal status $(P<0.001)$, oestrogen $(P=0.17)$ and progesterone receptors $(P=0.04)$ was developed. The nomogram accuracy was $87.3 \%$ ( $95 \%$ confidence interval $(C l)$, 84.45-90.2\%). Overall, the area under the ROC curve (AUC) was $86.1 \%$ for the validation set from the Institut Curie-René Huguenin, and $63.8 \%$ for the MD Anderson validation set. The negative predictive value (NPV) was high in the three cohorts (97-99\%).

Conclusions: We developed and validated a strong metastasis risk calculator that can evaluate with high accuracy an individual's risk of harbouring synchronous metastases at diagnosis of primary BC.

Condensed abstract: A nomogram to predict synchronous metastases at diagnosis of breast cancer was developed and externally validated. This tool allows avoiding unnecessary expensive work-up.

There were about 1384155 newly diagnosed breast cancer (BC) cases worldwide in 2008, as reported by the International Agency for Research on Cancer (IARC, 2008). A recent systematic analysis showed a $3.1 \%$ annual increase in $\mathrm{BC}$ incidence during the last three decades (Forouzanfar et al, 2011). In newly diagnosed cases, systematic body screening will be proposed to achieve accurate staging of the disease (UICC TNM) and to guide the primary treatment strategy (Edge et al, 2010). This work-up aims at identifying the subgroup of patients with distant metastases at diagnosis, who have a distinctly more severe prognosis. Data from the Surveillance Epidemiology and End Results (SEER) programme showed that synchronous distant metastases at diagnosis of primary BC are present in 5\% of patients (Howlader et al, 2011).

Accurate staging at diagnosis has clinical and psychological implications, such as the possible avoidance of mastectomy or of certain radiotherapy fields, of prolonged anti-cancer treatment or of anti-osteoclastic drugs. However, many expensive and pointless work-up examinations are performed in women with localised BC. The identification of clinical and tumour factors associated with synchronous metastases may therefore be useful to clinicians. The benefit of systematic body imaging is controversial, but it is very often proposed to all patients in the absence of specific markers of

*Correspondence: Dr C Mazouni; E-mail: chafika.mazouni@gustaveroussy.fr

Received 20 September 2014; revised 7 January 2015; accepted 12 January 2015; published online 10 February 2015

(c) 2015 Cancer Research UK. All rights reserved 0007-0920/15 
metastases (Carlson et al,1996; Chen et al, 2000; Gerber et al, 2003). The timing of complete staging is also debated, but clearly doing this evaluation before surgery is relevant because, although surgery is performed most of the time, whole body imaging can influence $\mathrm{BC}$ staging and clinical management, particularly in the case of oligometastatic disease. For instance, Yap et al (2001) showed that whole body FDG PET in BC altered the clinical stage in 36\% of patients and the clinical management in 60\%. The leading professional societies such as the American Society of Clinical Oncology (ASCO) or the European Society of Medical Oncology (ESMO) do not recommend extensive staging in localised BC, except in large tumours, or in the presence of clinically positive axillary lymph nodes.

Identifying high-risk groups would help decreasing the number of unnecessary exams. To date, no statistical model has been developed to predict the likelihood of synchronous metastases at diagnosis. In recent years, nomograms have gained popularity in clinics and have been proposed in oncology to address the individual-based prognosis (Kattan et al, 1998; Hanrahan et al, 2007; Mazouni et al, 2011). Nomograms are statistically based tools that provide the overall probability of a specific outcome for an individual patient. Factors associated with a defined event are incorporated in the nomogram and the calculated probability of the event occurrence is provided in graphical formats.

In this study, we developed a preoperative nomogram based on clinico-pathologic factors to predict the probability of synchronous distant metastases at diagnosis of primary BC. We then validated this nomogram in two independent data sets.

\section{PATIENTS AND METHODS}

Training population. The study population consisted of 2059 consecutive women who were diagnosed with primary BC at the One-Stop Unit of the Institut Gustave Roussy (IGR), Villejuif, France, between April 2004 and March 2010; 4461 consecutive patients from the Institut Curie-René Huguenin (CRH), SaintCloud, France and 2550 patients treated in the Breast Medical Oncology Department at the MD Anderson Cancer Center. All patients were enrolled over the same period after primary diagnosis of $\mathrm{BC}$ and irrespectively of the subsequent treatments (primary surgery and neo-adjuvant chemotherapy). All patients diagnosed with invasive $\mathrm{BC}$ at IGR and $\mathrm{CRH}$ underwent an initial standardised work-up that consisted in a clinical examination, blood tests including serum markers (CA 15-3 and/or CEA and/or CYFRA 21.1), bone scintigraphy, chest X-ray, abdominal and pelvic ultrasound. At MD Anderson Cancer Center, X-ray, thoraco-abdominal CT and bone scan were performed. Elevated tumour markers alone were not considered as a proof of metastases. The diagnosis of metastases was made based on typical radiological images. Tumour markers were measured and a focused biopsy was carried out in the case of isolated/uncertain lesion(s). The internal Institutional Review Boards of the three centres gave their approval for this study.

Statistical analysis. Variables evaluated at the time of the initial diagnosis included patient age (as a continuous variable), family history of BC, clinical (or radiological if non-palpable) tumour size (cT) (as a continuous variable), oestrogen receptor (ER) and progesterone receptor (PR) status (by immunohistochemical analysis), histological grade and HER2 status. Hormone receptor positivity was defined based on positive staining for ER and/or PR in at least $10 \%$ of cancer cell nuclei in France, and $\geqslant 5 \%$ at MD Anderson Cancer Center (MDACC). HER2 status was defined according to the ASCO guidelines. The ER, PR and HER2 status were entered as positive or negative.

The factors predictive for the presence of synchronous distant metastases at diagnosis were identified through univariate and multiple logistic regression analyses. Odds ratios were calculated to estimate the strength of the association between individual risk factors and synchronous metastases. Factors found to be significantly associated with synchronous metastases in the univariate analysis $(P<0.20)$ were included in the multivariate analysis.

Using the IGR data set, a logistic regression-based nomogram was developed to predict synchronous metastases at diagnosis. Backward elimination was performed to choose the covariates to be retained in the model. The discriminative power of the model was quantified in terms of discrimination and calibration. Discrimination was quantified by the area under the receiver-operating characteristic (ROC) curve (AUC) and 95\% confidence intervals (CIs) were estimated. In all, 200 bootstrap resamples were used for internal validation of the accuracy estimates and to reduce overfit bias. Sensitivity, specificity, and positive (PPV) and negative predictive values (NPV) and their 95\% CI were calculated for various cutoff points of the calculated risk score.

External validation was performed by using the nomogram for patients from the two independent validation cohorts. All statistical analyses were two-sided and were performed using the $\mathrm{R}$ cran Design package ( $\mathrm{R}$ Development Core Team, 2010). The characteristics of the three cohorts were compared using the Chi-square or Fisher exact tests for qualitative data and the Kruskal-Wallis test for quantitative data.

\section{RESULTS}

A total of 2059 patients diagnosed with primary BC at the OneStop Unit, IGR, over a 6-year period, were used to develop the nomogram. Among them, 23.7\% (488 out of 2059) received neoadjuvant treatment and $4.4 \%$ (91 out of 2059) had synchronous distant metastases at diagnosis.

Table 1 shows the univariate and multivariate analyses of potential predictors of synchronous metastases at diagnosis. Clinical tumour size $(\mathrm{cT})$, clinical nodal status $(\mathrm{cN}), \mathrm{ER}, \mathrm{PR}$ and

Table 1. Univariate and multivariate regression analyses

\begin{tabular}{|c|c|c|c|c|}
\hline & $\begin{array}{c}\text { Univariate } \\
\text { OR }(95 \% \mathrm{Cl})\end{array}$ & $P$-value & $\begin{array}{l}\text { Multivariate } \\
\text { OR }(95 \% \mathrm{Cl})\end{array}$ & $P$-value \\
\hline Age & $1.25(0.91-1.71)$ & 0.1702 & - & - \\
\hline Clinical tumour size (mm) & $1.94(1.72-2.20)$ & $<0.001$ & $1.7(1.5-1.9)$ & $<0.001$ \\
\hline Clinical nodal status & $7.8(5-12)$ & $<0.001$ & $4.1(2.6-6.6)$ & $<0.001$ \\
\hline Oestrogen receptor status & $0.66(0.41-1.07)$ & 0.09 & $1.7(0.9-3.4)$ & 0.13 \\
\hline Progesterone receptor status & $0.53(0.35-0.81)$ & 0.0035 & $0.6(0.3-1)$ & 0.05 \\
\hline Nuclear grade & & & $1.3(0.6-2.8)$ & 0.49 \\
\hline HER2 positive & $1.41(0.82-2.44)$ & 0.21 & - & - \\
\hline
\end{tabular}




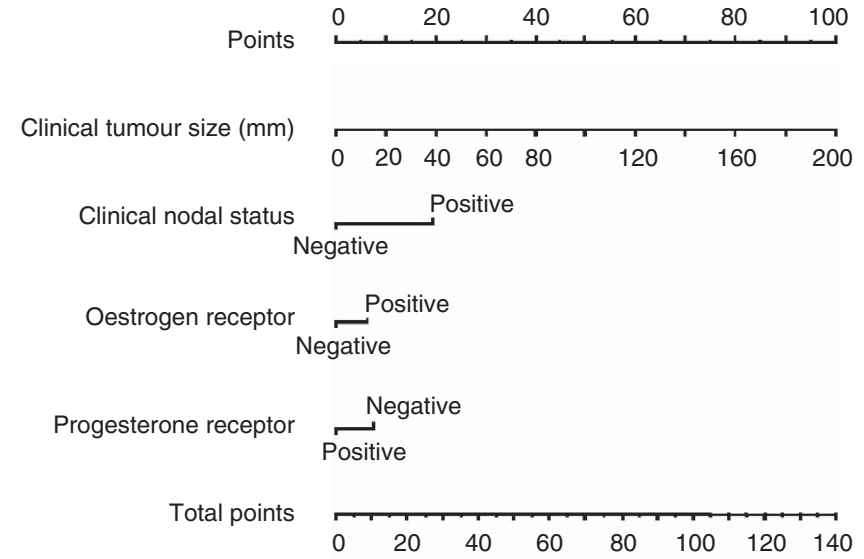

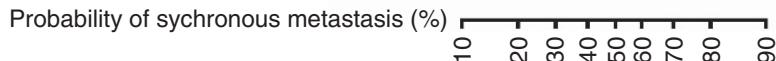

Figure 1. Nomogram based on 2059 patients treated at the Institut Gustave Roussy, for predicting the risk of synchronous metastases at first diagnosis of primary BC. To obtain the predicted probability of synchronous metastases, locate patient values at each axis. Draw a vertical line upward to the 'Points' axis to determine the points scored by the variable. Sum the points for all variables and locate the sum on the 'Total points' axis.

nuclear grade were significantly associated with the risk of synchronous metastases in the univariate analysis and, therefore, they were included in the multivariate analysis (Table 1). The variables used to develop the nomogram were clinical tumour size (cT, $P<0.001)$, clinical nodal status $(\mathrm{cN}, P<0.001)$, ER $(P=0.17)$ and PR $(P=0.04)$ (Figure 1). For instance, a woman with a $30-\mathrm{mm}$ tumour (15 points), negative $\mathrm{cN}$ status (0 point), ER positive (5 points) and PR negative ( 8 points) would score 28 points that can be converted in a $4 \%$ probability of having synchronous metastases at primary diagnosis.

The performance of the risk score to differentiate patients with or without metastases was assessed using the ROC analysis (Figure 2). The AUC was 87.3\% (95\% CI, 84.4-90.2\%). The nomogram predictions were then compared with the actual probability of having synchronous metastases in the 2059 women (Figure 3). The dashed line represents the performance of an ideal nomogram, where the predicted outcome would overlap perfectly with the actual outcome. The performance of the IGR nomogram is shown by the plotted solid line.

The nomogram was then validated externally using the $\mathrm{CRH}$ and MDACC data sets. The $\mathrm{CRH}$ cohort characteristics were comparable to those of the training IGR cohort, while those of the MDACC data set were significantly different, particularly cT, ER, PR and HER2 status and histological grade (Table 2). The rates of synchronous metastases were different between the IGR and CRH cohorts $(4.4 \%$ and $3.2 \% ; P=0.02)$, but not between the IGR and MDACC series ( $4.4 \%$ and $5.1 \% ; P=0.35$ ) (Table 2). In the $\mathrm{CRH}$ cohort, only 591 (13.2\%) patients received neoadjuvant treatment, while 2399 (94\%) of the MDACC patients received primary treatment. Figure 4 shows the distribution of the predicted probability of having synchronous metastases for each patient calculated with the nomogram. Most patients (Figure 4) with BC had a predicted metastatic risk lower than $10 \%$. The observed AUC of the constructed nomogram was $86.1 \%$ (95\% CI, 83.2-89.1\%) for the $\mathrm{CRH}$ validation cohort and $63.8 \%$ (95\%CI, 58.8-68.8\%) for the MDACC validation set (Figure 2). Therefore, the prediction model based on the IGR data overestimated the actual probability compared with the MDACC data. The respective sensitivity, specificity, NPV and PPV are shown in Table 3. Sensitivity and specificity were good

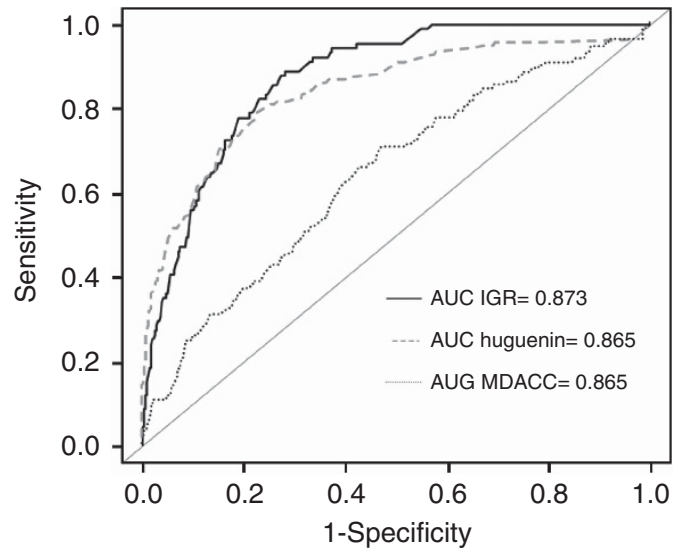

Figure 2. Receiver-operating characteristics curve for the performance of the risk score in identifying patients with synchronous metastases in the three data sets.

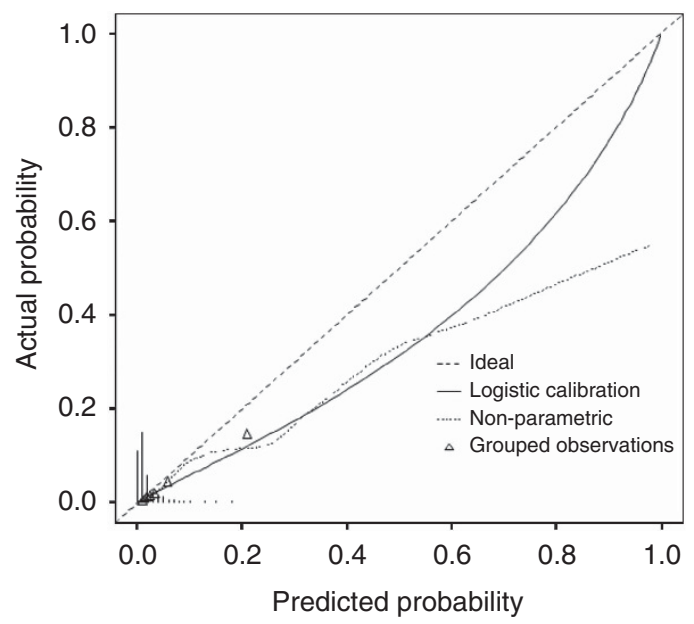

Figure 3. Calibration plot of the internal and external validation cohorts. The $x$ axis shows the prediction calculated using the nomogram, and the $y$ axis shows the observed rates of synchronous metastases for patients in the IGR cohort. The dashed line is the reference line, where an ideal nomogram would lie. The solid line indicates the performance of the IGR nomogram applied to the validation cohort. The solid line is not close to the dashed line of the ideal nomogram and is not always within the $10 \%$ margin of error.

for the IGR and CRH data sets and lower for the MDACC cohort. In all three cohorts, the NPV was higher than $96 \%$.

Finally, a computer program was developed to help physicians determine the risk of synchronous metastases at primary $\mathrm{BC}$ diagnosis. The program called 'synchronous metastases in $\mathrm{BC}$ ' is in Java. An internet browser with Java capability is required to run the applets. An example of a screen is shown in Figure 5. The applets will be freely available online through the IGR, CRH and MDACC websites.

Development of a nomogram based on the three cohorts. To correct for the lack of reproducibility and verify whether the same criteria were selected, we build a model that incorporated all patients from the three cohorts $(n=9070)$. In univariate analysis, clinical tumour size $(\mathrm{cT}, P<0.001)$, clinical nodal status $(\mathrm{cN}, P<0.001)$, ER $(P<0.001)$, PR $(P<0.001)$, nuclear grade $(P<0.001)$ and HER2 status $(P<0.001)$ were predictors of the metastatic status. In the final model, $\mathrm{cT}(P<0.001), \mathrm{cN}(P<0.001)$, PR $(P<0.001)$, nuclear grade $(P=0.01)$ and HER2 $(P=0.01)$ status were retained to build the nomogram. HER2 status could 
Table 2. Comparison of the baseline characteristics in the three study cohorts

\begin{tabular}{|c|c|c|c|c|c|}
\hline & $\begin{array}{c}\text { Training set } \\
\text { IGR } \\
n=2059\end{array}$ & $\begin{array}{c}\text { Validation set } 1 \\
\text { CRH } \\
n=4461\end{array}$ & $P^{*}$ value & $\begin{array}{c}\text { Validation set } 2 \\
\text { MDACC } \\
n=2550\end{array}$ & $P * \star$ value \\
\hline Age, median (range) & $60(23-96)$ & $58(21-95)$ & $<0.001$ & $50(19-91)$ & $<0.001$ \\
\hline $\begin{array}{l}\text { Clinical tumour size in } \mathrm{mm} \\
\text { median (range) }\end{array}$ & $16(2-200)$ & $15(0-200)$ & 0.91 & $35(0-200)$ & $<0.001$ \\
\hline Clinical node status (\%) & & & & & $<0.001$ \\
\hline $\begin{array}{l}\text { Negative } \\
\text { Positive }\end{array}$ & $\begin{array}{r}1693(82.2) \\
366(17.8)\end{array}$ & $\begin{array}{r}3750(84.1) \\
711(15.9)\end{array}$ & & $\begin{array}{r}858(33.6) \\
1692(66.4)\end{array}$ & \\
\hline Oestrogen receptor status (\%) & & & 0.65 & & $<0.001$ \\
\hline $\begin{array}{l}\text { Negative } \\
\text { Positive }\end{array}$ & $\begin{array}{r}400(19.4) \\
1659(80.6)\end{array}$ & $\begin{array}{r}890(20) \\
3571(80)\end{array}$ & & $\begin{array}{r}893(35) \\
1657(65)\end{array}$ & \\
\hline Progesterone receptor status (\%) & & & 0.96 & & $<0.001$ \\
\hline $\begin{array}{l}\text { Negative } \\
\text { Positive }\end{array}$ & $\begin{array}{r}741(36) \\
1318(64)\end{array}$ & $\begin{array}{l}1601(35.9) \\
2860(64.1)\end{array}$ & & $\begin{array}{l}1303(51.1) \\
1247(48.9)\end{array}$ & \\
\hline Tumour grade (\%) & & & $<0.001$ & & $<0.001$ \\
\hline $\begin{array}{l}1-2 \\
3\end{array}$ & $\begin{array}{r}1417(68.8) \\
642(31.2)\end{array}$ & $\begin{array}{l}3357(75.2) \\
1104(24.7)\end{array}$ & & $\begin{array}{r}921(36.1) \\
1629(63.9)\end{array}$ & \\
\hline HER2 (\%) & & & $<0.001$ & & $<0.001$ \\
\hline $\begin{array}{l}\text { Positive } \\
\text { Negative } \\
\text { Unknown } \\
\text { Synchronous metastases }\end{array}$ & $\begin{array}{r}260 / 1807(14.4) \\
1547 / 1807(85.6) \\
252(12.2) \\
91(4.4)\end{array}$ & $\begin{array}{r}492 / 4349(11.3) \\
3857 / 4349(88.7) \\
112(2.5) \\
144(3.2)\end{array}$ & 0.02 & $\begin{array}{c}382 / 1909(20) \\
1527 / 1909(80) \\
641(25.1) \\
129(5.1)\end{array}$ & 0.35 \\
\hline
\end{tabular}

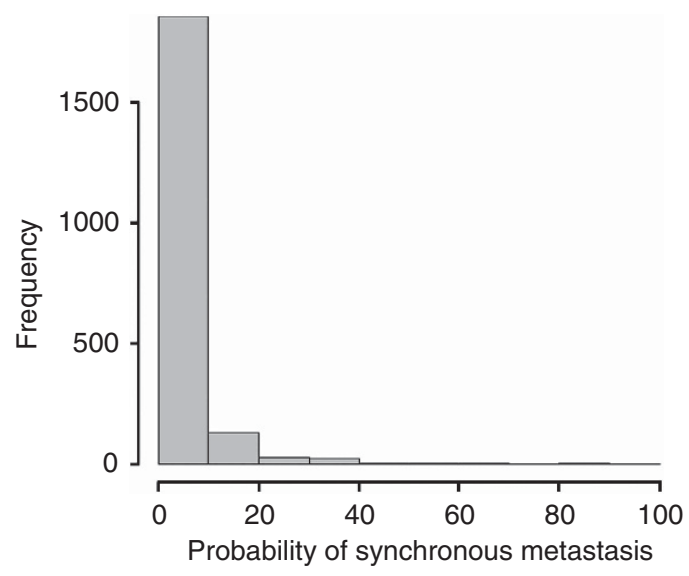

Figure 4. Distribution of predicted probability of population with synchronous metastases in all data sets. The $x$ axis of Figure 1 shows the prediction calculated using the nomogram, and the $y$ axis shows the observed rates of synchronous metastases for patients in the training cohort.

not be assessed in $11 \%$ of patients. The AUC for the constructed nomogram was $80.2 \%$ (95\% CI: $78-82.4 \%$ ). The sensitivity was $81.8 \%$, the specificity $65.3 \%$, the NPV $91 \%$ and the PPV was $1.2 \%$.

\section{DISCUSSION}

The estimation of the individual risk of synchronous metastases at diagnosis of primary $\mathrm{BC}$ is attractive because it can facilitate the decision regarding the initial staging work-up, thus avoiding pointless examinations. Several nomograms have been developed for patients with BC (Van Zee et al, 2003; Rouzier et al, 2005; Houvenaeghel et al, 2009). Most of these nomograms were developed to predict the nodal status or response to treatment.
Table 3. Sensitivity, specificity, PPV, and NPV for the risk calculator

\begin{tabular}{|c|c|c|c|c|}
\hline & $\begin{array}{c}\text { Sensitivity } \\
\text { (\%) }\end{array}$ & $\begin{array}{c}\text { Specificity } \\
\text { (\%) }\end{array}$ & NPV & PPV \\
\hline Training set (IGR) & 89 & 71.8 & $99.3(87.2)$ & $12.8(0.7)$ \\
\hline Validation set (CRH) & $81.9(82.6)$ & $76.1(75.7)$ & $99.2(89.8)$ & $10.3(0.8)$ \\
\hline Validation set (MDACC) & 70.5 & 52.8 & 97.1 & 7.4 \\
\hline \multicolumn{5}{|c|}{$\begin{array}{l}\text { Abbreviations: } \quad C R H=\text { Institut Curie-René Huguenin; IGR=Institut Gustave Roussy; } \\
\text { MDACC = MD Anderson Cancer Center; NPV= negative predictive value; PPV= positive } \\
\text { predictive value. }\end{array}$} \\
\hline
\end{tabular}

The present work is the first attempt to propose a diagnostic nomogram to improve BC staging by calculating the risk of initial distant metastases in patients with BC. The nomogram was validated internally and externally using three large data sets from reference cancer centres.

At present, in the presence of synchronous metastases, systemic treatment is proposed in addition to the local treatment of the primary tumour like for local tumours. The level of proof regarding local treatment is low, but the homogeneous results of several retrospective series support this practice (Ali and Le Scodan, 2011). However, mastectomy could be avoided in non-inflammatory cases. Local therapy of metastatic sites might be proposed, as well as bone-targeting treatments in the case of bone involvement.

The place of imaging in BC initial staging remains controversial. Currently, there is no evidence to support routine screening for metastatic disease in asymptomatic women with early operable BC (T1-2, N0-1), and this screening may not be cost effective (Gerber et al, 2003). Imaging for staging is inconsistently recommended by the different National Cancer Institutes worldwide (Ciatto et al, 1988; Harris, 2000; Myers et al, 2001). Most Cancer Societies recommend whole body staging only for large tumours or in the presence of positive nodes (Schnipper et al, 2012; Senkus et al, 2013). Previous studies on cost savings suggest focusing on metastasis screening by chest X-ray and blood tests alone in 

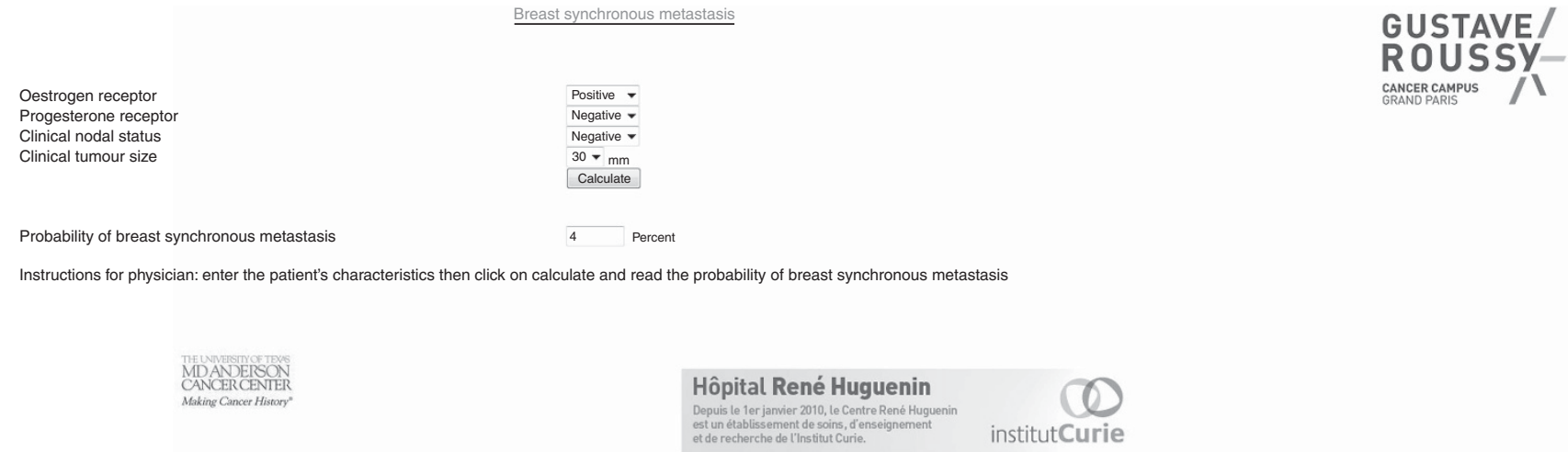

Figure 5. Example of a screen from the computer program 'synchronous metastases in breast cancer' that was developed from the nomogram described in this study to provide patients and physicians with information to assist them in treatment decision-making with regard to the risk of metastasis.

patients with stage I-II BC with less than five affected axillary lymph nodes (Norum and Andreassen, 2000; Ravaioli and Tassinari, 2000). However, this requires prior surgery. The broader knowledge we have today of tumour biology should be used to identify patients at high risk of metastatic dissemination, and to avoid unnecessary initial and subsequent screenings.

An interesting aspect of our nomogram is the inclusion of classic prognostic factors such as tumour size, nodal clinical, and hormone status. Our model combines independent factors that allow appraising the magnitude of the impact of each factor on the probability of synchronous metastases. The use of classic, wellestablished clinicopathological factors helps make the nomogram generalisable in the routine clinical practice. The probability of synchronous metastases in a patient is easily calculated by using the nomogram or the web interface. The practical application is the identification of high-risk women by using a combination of factors that together strongly increases the risk of metastases. An acceptable cutoff has yet to be defined with a balance between risk and cost saving. Clearly, most patients with $\mathrm{BC}$ have a predicted metastatic risk lower than $10 \%$ at diagnosis. We thus propose a definition of low-risk patients based on an observed metastatic risk lower than $4 \%$ in agreement with the rates we detected in the two European cohorts. This low threshold has to be prospectively evaluated and patients could be considered at high risk when showing a metastatic risk higher than $10 \%$.

We acknowledge that this study has some limitations. The multicentre cohorts used to validate this nomogram were heterogeneous. Thus, the metastasis rates were variable between centres, with the highest prevalence in the MDACC cohort. This was due to a significantly larger tumour size, higher nuclear grade, and frequent HR negativity in the MDACC population. As a consequence, the prediction model developed in the training set overestimated the actual probability in the MDACC cohort. For high predicted values, especially when higher than $20 \%$, the nomogram tends to overestimate the rate of synchronous metastases. While the two European cohorts were quite similar, the MDACC cohort included mostly patients who received neoadjuvant treatment as they were at higher risk for metastasis. Therefore, we also developed a nomogram based on all patients and in this final model, tumour grading and HER2 status significantly predicted the risk of synchronous metastasis. One limitation of this final model is the high number of patients without HER2 assessment and the absence of external validation. The observed AUC of this nomogram was lower (80.2\%) than the one of the nomogram based on the initial training cohort.

In the future, we plan to validate this preoperative nomogram using other data sets and in a prospective manner. This nomogram was based on classic clinico-pathological factors, but emerging biomarkers and genomic patterns could also be tested. It would be interesting to incorporate them into future nomograms to provide greater accuracy of risk estimation for distant metastases in the heterogeneous $\mathrm{BC}$ population.

In conclusion, estimation of the risk for distant metastases at primary diagnosis of $\mathrm{BC}$ may be helpful to personalise the decision-making strategy in $\mathrm{BC}$ patients. In this study, we developed a nomogram for clinical use to estimate this risk and avoid unnecessary expensive work-up in low-risk group which threshold could be $<4 \%$. Incorporating significant biological factors in future studies could improve the accuracy of this statistical model.

\section{ACKNOWLEDGEMENTS}

We thank Lorna Saint Ange and Elisabetta Andermarcher for editing.

\section{CONFLICT OF INTEREST}

The authors declare no conflict of interest.

\section{REFERENCES}

Ali D, Le Scodan R (2011) Treatment of the primary tumor in breast cancer patients with synchronous metastases. Ann Oncol 22: 9-16.

Carlson RW, Goldstein LJ, Gradishar WJ, Lichter AS, McCormick B, Moe RE, Theriault RL (1996) NCCN breast cancer guidelines: the National Comprehensive Cancer Network. Oncology 10: 47-75.

Chen EA, Carlson GA, Coughlin BF, Reed Jr WP, Garb JL, Frank JL (2000) Routine chest roentgenography is unnecessary in the work-up of stage I and II breast cancer. J Clin Oncol 18: 3503-3506.

Ciatto S, Pacini P, Azzini V, Neri A, Jannini A, Gosso P, Molino A, Capelli MC, di Costanzo F, Pucciatti MA (1988) Preoperative staging of primary breast cancer: a multicentric study. Cancer 61: 1038-1040.

Edge SB, Byrd DR, Compton CC, Fritz AG, Greene FL, Trotti A (eds) (2010) AJCC Cancer Staging Manual, 7th edn. Springer: New York.

Forouzanfar MH, Foreman KJ, Delossantos AM, Lozano R, Lopez AD, Murray CJ, Naghavi M (2011) Breast and cervical cancer in 187 countries between 1980 and 2010: a systematic analysis. Lancet 378: 1461-1484.

Gerber B, Seitz E, Müller H, Krause A, Reimer T, Kundt G, Friese K (2003) Perioperative screening for metastatic disease is not indicated in patients with primary breast cancer and no clinical signs of tumor spread. Breast Cancer Res Treat 82: 29-37.

Hanrahan EO, Gonzalez-Angulo AM, Giordano SH, Rouzier R, Broglio KR, Hortobagyi GN, Valero V (2007) Overall survival and cause-specific 
mortality of patients with stage T1a,bN0M0 breast carcinoma. J Clin Oncol 25: 4952-4960.

Harris JR (2000) Staging of breast cancer. In Diseases of the Breast, Harris JR, Lippman ME, Marrow M, Osborne CK (eds) 2nd edn, pp 403-406. Lippincott, Williams and Wilkins: Philadelphia.

Houvenaeghel G, Nos C, Giard S, Mignotte H, Esterni B, Jacquemier J, Buttarelli M, Classe JM, Cohen M, Rouanet P, Penault Llorca F, Bonnier P, Marchal F, Garbay JR, Fraisse J, Martel P, Fondrinier E, Tunon de Lara C, Rodier JF (2009) A nomogram predictive of non-sentinel lymph node involvement in breast cancer patients with a sentinel lymph node micrometastasis. Eur J Surg Oncol 35: 690-695.

Howlader N, Noone AM, Krapcho M, Garshell J, Miller D, Altekruse SF, Kosary CL, Yu M, Ruhl J, Tatalovich Z, Mariotto A, Lewis DR, Chen HS, Feuer EJ, Cronin KA (eds) (2011) SEER Cancer Statistics Review, 1975-2008. National Cancer Institute: Bethesda, MD. http://seer. cancer.gov/csr/1975_2008/. Based on November 2010 SEER data submission, posted to the SEER website.

International Agency for Research on Cancer. GLOBOCAN (2008) Cancer incidence and mortality worldwide. http://globocan.iarc.fr/ globocan2008.htm.

Kattan MW, Eastham JA, Stapleton AM, Wheeler TM, Scardino PT (1998) A preoperative nomogram for disease recurrence following radical prostatectomy for prostate cancer. J Natl Cancer Inst 90: 766-771.

Mazouni C, Romain S, Bonnier P, Martin PM (2011) A nomogram predicting the probability of primary breast cancer survival at 2- and 5-years using pathological and biological tumor parameters. J Surg Oncol 103: 746-750.

Myers RE, Johnston M, Pritchard K, Levine M, Oliver T (2001) Baseline staging tests in primary breast cancer: a practice guideline. CMAJ 164: 1439-1444.

Norum J, Andreassen T (2000) Screening for metastatic disease in newly diagnosed breast cancer patients. What is cost-effective? Anticancer Res 20: 2193-2196.
R Development Core Team (2010) R: A language and environment for statistical computing. R Foundation for Statistical Computing, Vienna, Austria. ISBN 3-900051-07-0, http://www.R-project.org/.

Ravaioli A, Tassinari D (2000) Staging of breast cancer: recommended standards. Ann Oncol 11: 3-6.

Rouzier R, Pusztai L, Delaloge S, Gonzalez-Angulo AM, Andre F, Hess KR, Buzdar AU, Garbay JR, Spielmann M, Mathieu MC, Symmans WF, Wagner P, Atallah D, Valero V, Berry DA, Hortobagyi GN (2005) Nomograms to predict pathologic complete response and metastasis-free survival after preoperative chemotherapy for breast cancer. J Clin Oncol 23: 8331-8339.

Schnipper LE, Smith TJ, Raghavan D, Blayney DW, Ganz PA, Mulvey TM, Wollins DS (2012) American Society of Clinical Oncology identifies five key opportunities to improve care and reduce costs: the top five list for oncology. J Clin Oncol 30: 1715-1724.

Senkus E, Kyriakides S, Penault-Llorca F, Poortmans P, Thompson A, Zackrisson S, Cardoso F (2013) ESMO Guidelines Working Group. Primary breast cancer: ESMO Clinical Practice Guidelines for diagnosis, treatment and follow-up. Ann Oncol 24(Suppl 6): vi7-23.

Van Zee KJ, Manasseh DM, Bevilacqua JL, Boolbol SK, Fey JV, Tan LK, Borgen PI, Cody 3rd HS, Kattan MW (2003) A nomogram for predicting the likelihood of additional nodal metastases in breast cancer patients with a positive sentinel node biopsy. Ann Surg Oncol 10: 1140-1151.

Yap CS, Seltzer MA, Schiepers C, Gambhir SS, Rao J, Phelps ME, Valk PE, Czernin J (2001) Impact of whole-body 18F-FDG PET on staging and managing patients with breast cancer: the referring physician's perspective. J Nucl Med 42: 1334-1337.

This work is published under the standard license to publish agreement. After 12 months the work will become freely available and the license terms will switch to a Creative Commons AttributionNonCommercial-Share Alike 4.0 Unported License. 